\title{
Measurement of residual stress of magnetic substance by surface acoustic waves
}

\author{
Hiroshi Osada, ${ }_{1}^{1, a)}$ Takumi Yamaguchi, ${ }^{2}$ Satoshi Nakamura, ${ }^{1}$ Hiroshi Hatafuku, ${ }^{1}$ \\ Shigeki Chiba, ${ }^{1}$ and Hideo Oka ${ }^{1}$ \\ ${ }_{1}^{1}$ Department of Electrical and Electronic Engineering, Faculty of Engineering, Iwate University, Morioka, \\ Iwate 020-8550, Japan \\ ${ }^{2}$ Tohoku Ricoh, Shibatamachi, Miyagi 989-1695, Japan
}

(Presented on 6 November 2007; received 7 September 2007; accepted 11 October 2007; published online 22 January 2008)

\begin{abstract}
In magnetic substances under oscillating stress due to the application of acoustic waves (such as ultrasonic waves), an oscillating anisotropic field may be generated by the inverse effect of magnetostriction. If this magnetic field oscillation can be detected, the stress state inside the magnetic substance can be determined. Based upon this principle, we have set up an apparatus for stress measurements, applied it to Ni plates with different residual stresses, and have obtained output signals corresponding to different stresses. This measuring technique makes it possible to know the stress state in structures before the formation of cracks, and hence offers a very promising nondestructive testing device. (C) 2008 American Institute of Physics. [DOI: 10.1063/1.2830641]
\end{abstract}

\section{INTRODUCTION}

Long-term usage of structures is very beneficial, in view of both economics and environmental considerations. In order to realize such long-term usage, structural safety must be checked periodically by nondestructive testing.

As nondestructive testing methods for structures, the pulsed ultrasonic method and the electromagnetic induction method are usually used to measure the position and size of flaws in structures. ${ }^{1-3}$ However, structural security hazards will be detected much earlier if the fatigue level of the structure can be measured before cracking.

When a magnetic substance has internal residual stress, the magnetoelastic energy is localized according to the magnitude of the stress and produces a magnetic field. Although this magnetic field is very small, it has been shown in a previous study ${ }^{4}$ that induced electromotive force (emf) is generated corresponding to the magnitude of the magnetic field due to the residual stress. A high-frequency magnetic field has been generated by the inverse effect of magnetostriction by applying ultrasonic waves to specimens. Using the induced emf generated by this method, it should be possible to measure the fatigue level of structural building materials containing magnetic substances such as steel before crack formation.

\section{PRINCIPLE OF MEASUREMENTS}

When a surface acoustic wave, such as those used in ultrasonication, is applied to the surface of an object, the longitudinal wave propagates through the object forming compressive-tensile regions inside. In magnetic materials such as steel, magnetoelastic energy is generated by the inverse effect of magnetostriction in compressive-tensile regions and an anisotropic magnetic field is induced. For pla-

\footnotetext{
${ }^{a)}$ Electronic mail: osada@iwate-u.ac.jp.
}

nar magnetic specimens, it is expected that the magnetic flux generated by the magnetic moment due to the anisotropic field will leak from the surface of specimens.

Figure 1 illustrates the magnetic moments on the surface of a specimen, through which a longitudinal wave is propagating. The magnetic moment $H_{\sigma}$ due to the anisotropic magnetic field induced by magnetoelastic energy from ultrasonic waves rotates depending on the sign of magnetostriction $\lambda$ and stress $\sigma$. For $\lambda>0, H_{\sigma}$ lines up perpendicularly in the compressive region $(\sigma<0)$ or parallel in the tensile region $(\sigma>0)$ to the direction of wave propagation. $H_{\sigma}$ at a given location in a specimen thus oscillates according to the propagation of the applied wave. The total sum of magnetic moments can be observed with a sensing coil as a change in the oscillating magnetic field. The magnitude of $H_{\sigma}$ is estimated to be very small, but it can readily be measured, since the induced emf increases in proportion to the frequency of ultrasonic waves.

\section{EXPERIMENTAL RESULTS AND DISCUSSION}

Based on the foregoing discussion, the induced emf was measured for Ni plates with different residual stresses.

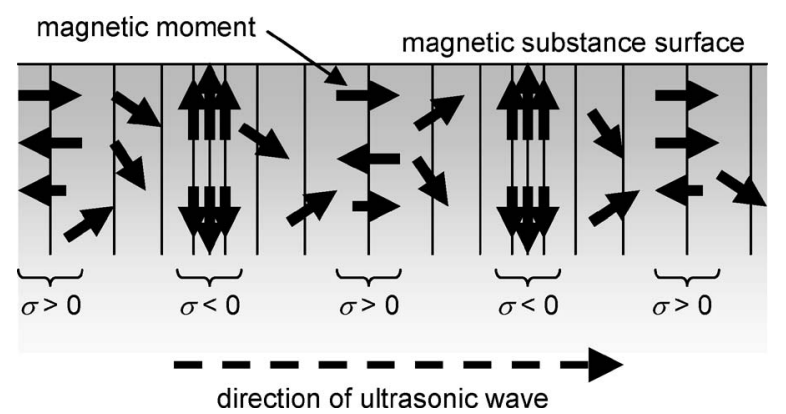

FIG. 1. Model for stresses and magnetic moments in a specimen under propagation of a longitudinal wave 

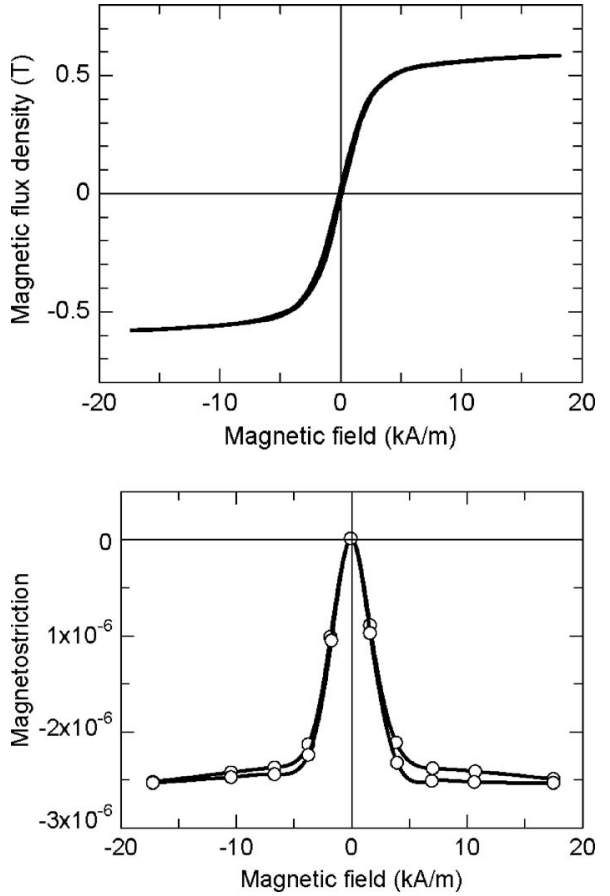

FIG. 2. B-H curve (a) and magnetostriction curve (b) for a Ni rod.

Figure 2 shows the $B$ - $H$ curve (a) and the magnetostriction curve (b) for a Ni rod sample after removing the residual stress by annealing. The $B-H$ curve shows the induced electromotive force, measured with a flux meter, that was generated in the coil wound around the sample when a magnetic field was applied with an external electromagnet. Moreover, the magnetostriction curve shows the measured resistance change of the strain gauge attached to the sample (KFN2350-D20-11, Kyowa) as the magnetic field was applied with the electromagnet. The resistance was measured using a Wheatstone bridge. This figure shows that the magnetostriction of $\mathrm{Ni}$ was saturated with the applied field as the $B-H$ curve was saturated.

\section{A. Experimental method}

Figure 3 shows a schematic diagram of the experimental apparatus, composed of an ultrasonic transducer, a power supply for magnetization, a coil for the bias magnetic field, a square wave pulse generator, a sensing coil, and a signal amplifier. A longitudinal ultrasonic wave was generated from the ultrasonic transducer by signals from the pulse generator,

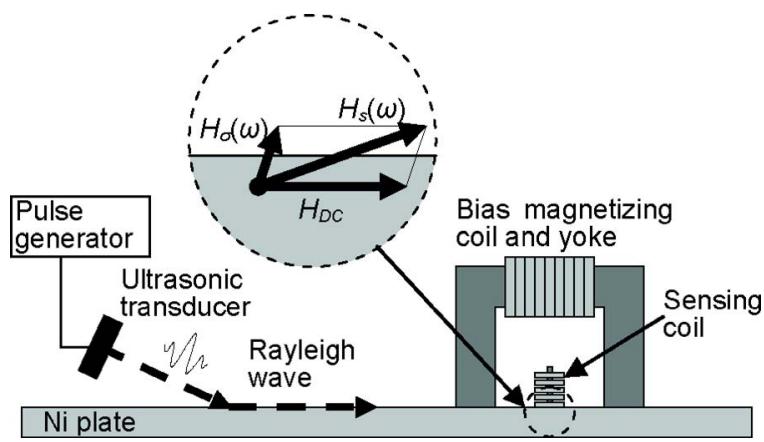

FIG. 3. (Color online) Schematic diagram of experimental apparatus.

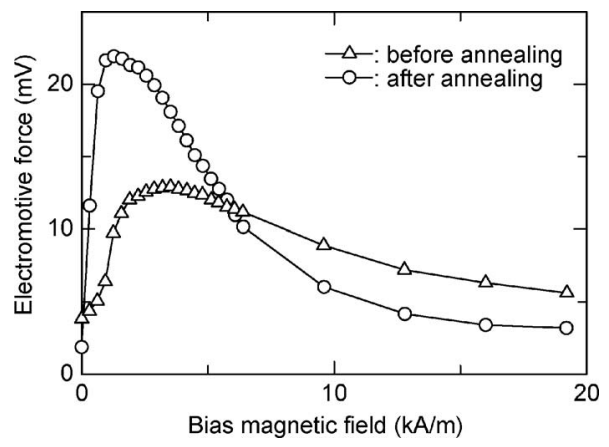

FIG. 4. Bias magnetic field vs induced emf curve for a Ni plate before and after annealing.

and was incident on the specimen at an angle of $60^{\circ}$ from the normal. The incident longitudinal ultrasonic wave propagated on the surface of the specimen as a Rayleigh wave accompanying a transverse wave. The induced emf due to propagating waves was measured with a sensing coil. The sensing coil was composed of a ferrite core of $1 \mathrm{~mm}$ in diameter and $6 \mathrm{~mm}$ in length and a wire of 600 turns. The separation between the ultrasonic transducer and the sensing coil was $70 \mathrm{~mm}$.

At the measuring point, the ac anisotropy magnetic field $H_{\sigma}(\omega)$ occurs by ac stress $\sigma(\omega)$ by an ultrasonic wave (angular frequency, $\omega)$. The following formulas define $H_{\sigma}(\omega)$ as a function of $\sigma(\omega){ }^{5}$

$$
H_{\sigma}(\omega)=\frac{-3 \lambda \sigma(\omega) \cos ^{2} \phi}{I},
$$

where $I$ is magnetization and $\phi$ is an angle of $\sigma$ and $I$.

$H_{\sigma}(\omega)$ is compounded with the dc bias magnetic field $H_{\mathrm{dc}}$, and forms the magnetic field $H_{S}(\omega)$. Therefore, the induced emf by $H_{\sigma}(\omega)$ in the measuring point changes depending on $\omega, H_{\sigma}(\omega)$, and $H_{\mathrm{dc}}$. If $\omega$ and $H_{\mathrm{dc}}$ are known, the stress state of an observation point can be estimated from the induced emf.

\section{B. Experimental results}

Ni plates (length, $300 \mathrm{~mm}$; width, $40 \mathrm{~mm}$; thickness, $3 \mathrm{~mm}$ ) were used as specimens for measurements. The sensing coil was placed at the center of each specimen. The frequency of the ultrasonic wave was $1 \mathrm{MHz}$.

Figure 4 shows the relation between the bias magnetic field and emf for a specimen before and after heat treatment. The annealing effect is clearly seen in the figure. The curve before heat treatment shows a smaller peak and a larger value of bias field at the peak position, in comparison with the curve after heat treatment. In each case the specimen was demagnetized before measurements, and consequently it is suggested that the observed difference is caused by the presence and absence of residual stress. In general, specimens before heat treatment have residual stresses, and stresses are removed by heat treatment. It is predicted therefore that the peak of the induced emf decreases and shifts toward higher bias values with increasing residual stresses.

Figure 5 shows the bias magnetic field versus induced emf characteristics for Ni plates heat treated and then rolled 


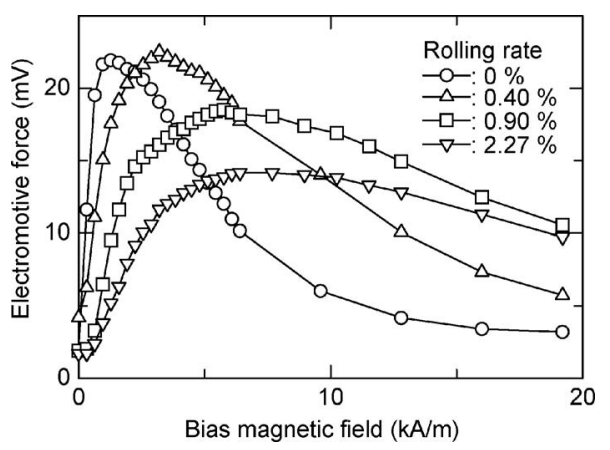

FIG. 5. Bias magnetic field vs induced emf curves for Ni plates with different rolling rates.

at different rolling rates $(0 \%-2.27 \%)$ to increase the residual stress gradually. The figure shows that the induced emf varies with the bias magnetic field as a function of rolling rate. The peak value of induced emf decreases with increases in the rolling rate, while the value of bias magnetic field at the peak increases. This shows that the observed change is due to the increase of residual stress by rolling.

\section{CONCLUSIONS}

A nondestructive testing method using the inverse effect of magnetostriction generated by application of ultrasonic waves has been proposed, which enables us to measure the stress state in planar specimens with a relatively simple apparatus. To measure the stress state in structures by this nondestructive method and to predict possible failure regions should be a very useful technique. The present apparatus will be also useful as a simple system to measure the distribution of magnetic moments in planar specimens.

${ }^{1}$ J. Blitz and G. Simpson, Ultrasonic Methods of Non-Destructive Testing (Chapman and Hall, London, 1996), p. 5.

${ }^{2}$ B. P. C. Rao and T. Jayakumar, J. Non-Destruct. Test. Eval. 2, 23 (2002).

${ }^{3}$ A. Sophian, G. Y. Tian, and S. Zairi, Sens. Actuators, A 125, 186 (2006).

${ }^{4}$ H. Hatafuku, C. Sarudate, and A. Konno, IEEE Trans. Magn. 38, 3309 (2002).

${ }^{5}$ S. Chikazumi, Physics of Ferromagnetism, 2nd ed. (Oxford, New York, 1997), p. 377. 